\title{
La fotografía y el fotoperiodismo, un objeto de estudio emergente en la investigación en comunicación en España
}

\section{Photography and photojournalism, an emerging object of study in communication research in Spain}

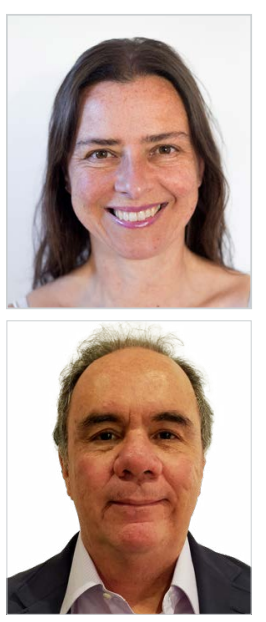

María Yanet Acosta Meneses. Licenciada y Doctora en Periodismo por la Universidad Complutense de Madrid (UCM) y posgrado en la Universidad de Columbia de Nueva York. Actualmente es Profesora ayudante doctor en la Universidad Rey Juan Carlos de Madrid y directora del Master de Comunicación y Periodismo Gastronómico The Foodie Studies. Es investigadora del grupo Mapcom (financiado en la convocatoria de Proyecto de I+D 2018) y de la cátedra UNESCO de Investigación en Comunicación de la URJC.

Universidad Rey Juan Carlos, España

myanet.acosta@urjc.es

ORCID: 0000-0003-1151-9863

Carlos Lozano Ascencio. Doctor en Ciencias de la Información por la Universidad Complutense de Madrid y Licenciado en Ciencias de la Comunicación por la Universidad Nacional Autónoma de México. Es profesor Titular de Universidad de Periodismo en la Universidad Rey Juan Carlos. En la actualidad dirige el Máster Universitario en Periodismo Cultural y Nuevas Tendencias de la URJC. Coordina la Sección: Teorías y métodos de investigación en Comunicación de la Asociación Española de Investigación en Comunicación (AE-IC). Es Co-IP del proyecto I+D “Mapas de la Investigación en Comunicación en las universidades españolas de 2007 a 2018" (MapCom). Miembro del grupo de investigación de excelencia Mediación Dialéctica de la Comunicación Social de la UCM, del Grupo Universitario de Mediación y Comunicación de la URJC y de la Cátedra COMUNESCO de Investigación en Comunicación de la URJC. Sus principales líneas de investigación: Comunicación de Riesgo y Catástrofes, Meta-investigación de la Comunicación e Historia del Periodismo.

Universidad Rey Juan Carlos, España

carlos.lozano@urjc.es

ORCID: 0000-0003-2537-0799

Recibido: 30/05/2021 - Aceptado: 19/10/2021 - En edición: 27/10/2021 - Publicado: 01/12/2021

\section{Resumen:}

Este artículo aborda la fotografía y el fotoperiodismo como objeto de estudio en la investigación en comunicación en las universidades españolas entre 2007 y 2018. De los documentos analizados se extrae que, pese a tratarse de un estudio emergente, por su interés social en la era de la posverdad, la producción científica vinculada a proyectos I+D+i no es aún relevante. Así, las temáticas que se han estudiado en estos doce años muestran el progresivo interés por investigar temas de relevancia internacional en las Ciencias Sociales como las identidades.
Received: 30/05/2021 - Accepted: 19/10/2021 - Early access: 27/10/2021 - Published: 01/12/2021

\section{Abstract:}

This paper addresses photography and photojournalism as an object of study in communication research in Spanish universities between 2007 and 2018. The documents analysed show that despite being an emerging study, due to the social interest it inspires in the era of post-truth, there is still relatively little scientific production linked to RDI projects. However, the topics that have been studied in these twelve years show an increasing interest in researching topics of international relevance in the Social Sciences, such as identities. It is worthy of note that many

Cómo citar este artículo:

Acosta Meneses, M. Y. y Lozano Ascencio, C. (2021). La fotografía y el fotoperiodismo, un objeto de estudio emergente en la investigación en comunicación en España. Doxa Comunicación, 33, pp. 393-404.

https://doi.org/10.31921/doxacom.n33a866 
Cabe destacar que aún muchos de estos trabajos, especialmente tesis doctorales, se ciñen al papel de la imagen en medios off-line y la mayoría de ellos son descriptivos. Pero se abre una nueva etapa para la investigación en comunicación en la que lo que ha sido una debilidad, la falta de una metodología clara de estudio, puede ser una fortaleza, dado que la fotografía puede ser interpretada desde diferentes enfoques y disciplinas en sintonía con las nuevas formas de pensar y de investigar estas disciplinas y que tienden hacia lo holístico. Se constata un gran interés por el desarrollo de este objeto de estudio que es clave en el panorama mediático mundial.

\section{Palabras clave:}

Fotografía; fotoperiodismo; comunicación; metainvestigación en comunicación; tesis doctorales; artículos científicos; comunicaciones en congresos. of these studies, especially doctoral theses, still adhere to the role of the image in offline media and most of them are descriptive. But a new era is opening for communication research in which what has previously been a weakness, the lack of a clear study methodology, may become a strength, given that photography can be interpreted from different approaches and disciplines in tune with new ways of thinking about and investigating these disciplines, these new approaches tending towards the holistic. There is evidence of great interest in the development of this object of study, which is considered a key element in the global media panorama.

\section{Keywords:}

Photography; photojournalism; communication; meta research in communication; doctoral theses; academic papers; conference proceedings.

\section{Introducción}

Que el Premio Pulitzer 2020 de fotografía recayera en el fotoperiodista español Emilio Morenatti ha venido a fortalecer la visibilidad del fotoperiodismo en España. La atención mediática a los trabajos fotoperiodísticos, las entrevistas en medios de comunicación a profesionales de este ámbito, charlas y proyectos de crowdfunding o de responsabilidad social están directamente relacionados con esta disciplina periodística. Entre los profesionales del fotoperiodismo en España de fama internacional podemos destacar a las tres fotógrafas adscritas a la prestigiosa Agencia Magnum -Cristina García Rodero, Cristina de Middel y Lúa Ribeiro-, los fotoperiodistas premiados con el Pulitzer como Javier Bauluz (1995), Manu Brabo (2013), Susana Vera (2020) y Emilio Morennatti (2021), así como, por ejemplo, Arturo Rodríguez y Samuel Aranda galardonados por los World Press Photo. A ellos se suma el reconocido trabajo de fotoperiodistas en activo como Maysun, Anna Surinnyach, Olmo Calvo y Judith Prat, entre otros muchos.

A esta visibilización pública también ha contribuido el recién estrenado programa en La 2 de RTVE "Detrás del instante" en el que cada episodio habla de un fotógrafo, muchos de ellos fotoperiodistas, como es el caso de Xurxo Lobato, Gervasio Sánchez, Raúl Cancio, César Lucas, Sandra Balsells, Manuel Outumuro e Isabel Azkárate.

A este programa se suma la popularidad de algunos documentales sobre fotoperiodistas españolas como Joana Biarnés, distribuido a través de la plataforma Filmin, así como exposiciones a través de festivales anuales de gran prestigio como PhotoEspaña (ya por su XXIV edición) o, incluso, la anual de los World Press Photo que se ha realizado en los últimos años de forma itinerante en Madrid y en Barcelona (excepto en 2020 debido a la pandemia provocada por la COVID-19).

En este momento en España coinciden varias generaciones de importantes fotoperiodistas, de manera que casi podríamos hablar de una edad de oro del fotoperiodismo en nuestro país. Sin embargo, esta profesión está sufriendo con crudeza la crisis financiera de los medios de comunicación tradicionales, por lo que hasta se ha llegado a hablar de su declive (Alcaide, 2017). 
Esta realidad no ha sido pasada por alto por los principales medios académicos y son estos temas principalmente los que han ocupado muchas de las publicaciones más recientes sobre fotoperiodismo en las revistas científicas españolas. Algunos de los últimos trabajos en ese sentido analizan las narrativas de la guerra y la paz (Baron Pulido, 2020), el imaginario del fake audiovisual (Cerdán Martínez \& Padilla Castillo, 2019), verificación de bulos (Guallar et al., 2020) , la construcción fotográfica de la realidad (Leon et al., 2018) la manipulación (Mayoral et al., 2019) y la imagen de la pobreza (Del Prado Flores et al., 2020)

A este interés temático se suma la incorporación del fotoperiodismo como asignatura en los planes docentes de muchas facultades de comunicación y de ciencias de la información de las universidades españolas tanto públicas como privadas que aún no lo habían incluido, tal y como se puede rastrear en el Registro de Universidades, Centros y Títulos (RUCT). Si buscamos además el plan docente de esta asignatura en muchas de las facultades que se imparte, se puede observar que el contenido no está destinado solo a entrenar al alumnado, sino a formarlo. Esto significa que, aunque a primera vista pueda parecer una asignatura eminentemente práctica -de hecho, en muchas ocasiones son fotoperiodistas profesionales los que imparten la docencia como profesorado asociado-, va más allá, pues tanto la teoría, como la historia, la educación de la mirada y el análisis de la imagen son claves en las competencias que debe adquirir el alumnado que decida cursarla.

El profesorado que en los últimos años ha impartido esta asignatura en diversos centros donde anteriormente no existía se está especializando y es por ello que consideramos que, de esta manera, la fotografía y el fotoperiodismo se dibujan también como una tendencia de interés científico en alza en el ámbito académico de la comunicación.

A esto se suma como motivos de una evidente evolución del interés de los investigadores e investigadoras en este objeto de estudio que se suman así a los investigadores ya consolidados de esta área como Margarita Ledo (Baeza, 2000), la aplicación de una metodología innovadora para su estudio como el framing, además de su indiscutible importancia en este momento social en la era de la postverdad.

Analizar lo que se ha investigado hasta ahora sobre el fotoperiodismo y la fotografía en comunicación y conectar los puntos que han dibujado este perfil investigador durante una década es lo que se propone en este trabajo como una parte crucial para poder afrontar tanto las debilidades de esta área de conocimiento como sus fortalezas.

Entre sus debilidades, la más discutida en el ámbito de la investigación es la escasa contribución de trabajos sobre esta temática en la producción científica universitaria. Sin embargo, considerando los datos obtenidos para este estudio a través de los resultados de los proyectos de I+D denominados de forma genérica como MapCom, las líneas trabajadas revelan que se trata de un objeto de estudio de interés creciente.

\section{Marco conceptual}

La preocupación por la falta de atención a la investigación académica en fotografía y la falta de consideración de esta disciplina artística, comunicativa y documental en el ámbito universitario ha sido expuesta desde los años 80 del siglo XX hasta la actualidad. Entre los investigadores que trabajaron este aspecto se encuentra Joan Fontcuberta con sus Notas sobre la fotografía espa- 
ñola de 1983 para la Historia de la Fotografía de Beaumont Newhall a la que siguió, en 1989, La fotografía y sus posibilidades documentales. Una introducción a su utilización en las Ciencias Sociales firmada por Bernardo Riego, Marie-Loup Souguez y Miguel Ángel Sánchez. Así lo refiere la más reciente investigación titulada “La investigación académica sobre fotografía en España” (García-Ramos \& Jiménez-Gómez, 2020). El artículo analiza las tesis doctorales sobre fotografía en la última década en las facultades de Humanidades y Ciencias Sociales de las universidades españolas. En él se señala la falta de especialización del profesorado y la dispersión del estudio como gran dificultad para su impulso, pero por otro lado se destaca la gran versatilidad de este objeto de estudio y lo que además lo sitúa en una posición privilegiada para afrontar estudios multidisciplinares.

A una conclusión similar llega el trabajo recientemente publicado sobre fotografía en las revistas científicas de impacto sobre documentación, que destaca que solo el 2,18\% del total aborda esta temática. Una cifra escasa "si tenemos en cuenta la ingente cantidad de documentos fotográficos que se conservan en archivos, bibliotecas, museos, bancos de imágenes, agencias y centros de documentación de las instituciones públicas y privadas, susceptibles de ser tratados y analizados para su uso y aplicación" (Sánchez-Vigil et al., 2021). El artículo critica la escasa relevancia de aspectos como el tratamiento de los fondos fotográficos en forma y fondo, digitalización, conservación y restauración dentro de la enseñanza superior y constata que las universidades con asignaturas y grados afines a esta disciplina constituyen el principal núcleo generador de investigación y de artículos.

Uno de los motivos que se pueden aducir para comprender esta falta de interés académico por esta área de conocimiento podría ser la falta de una metodología clara para acometer el análisis de la imagen. Sin embargo, tal y como resalta quien se ha convertido en referente teórico de la investigación fotográfica en España, Javier Marzal Felici:

"La Teoría de la Imagen no puede aspirar, en ningún caso, a desarrollar un cuerpo de conocimiento con capacidad de modelización matemática predictiva, ni posee un objeto de estudio estable o nítidamente definido desde un único paradigma científico" (MarzalFelici, 2021).

Esta observación parte de la subjetividad con la que el clásico teórico de la imagen Roland Barthes relacionó el análisis de una fotografía (Barthes, 2007) y con las diversas lecturas ofrecidas por otra de las referentes tradicionales en este tipo de investigaciones (Sontag, 2007) y se relaciona con otro referente español para abordar la era de la postfotografía como es Fontcuberta (Fontcuberta, 2016).

No obstante, el interés científico por el análisis fotográfico lejos de decaer, ha sido alentado por metodologías como la del framing (Ardevol-Abreu, 2015) entre cuyas investigaciones destacadas se sitúa "Visually framing the invasion and occupation of Iraq in Time, Newsweek, and US News \& World Report" (Schwalbe, 2013) que sirvió de inspiración del estudio realizado en España "Análisis de contenido de la representación fotográfica de la crisis de los refugiados sirios y su incidencia en el framing visual" (Del Ramo, 2016) y otros estudios más recientes como el publicado sobre los refugiados en la prensa europea (Amores et al., 2019).

Además, hay otras las metodologías que se utilizan en la interpretación de la imagen desde diversas perspectivas de estudio, como la antropológica, la documental, la semiótica, la histórica, la iconológica, biográfica, ética, etc. y es que se puede decir que “cualquier propuesta metodológica, en la práctica, es una teoría de la imagen o de la representación" (Marzal-Felici, 2021). 


\section{Metodología}

En la presente investigación nos centramos en el análisis bibliométrico de la investigación en fotografía y fotoperiodismo en el área de Comunicación entre 2007 y 2018 (por ser el periodo de estudio del grupo de investigación del que parte esta investigación), tomando en cuenta las tesis doctorales del área, los artículos científicos publicados en las principales publicaciones españolas de la época analizada atendiendo a su índice de impacto y siempre que tuvieran una vinculación directa y expresa con un proyecto estatal y financiado de investigación y desarrollo y las comunicaciones presentadas y publicadas en el libro de actas de los congresos de la Asociación Española de Investigación en Comunicación. Lo que significa que estos datos representan la investigación de alto rendimiento en España.

Estos datos se extraen de dos proyectos I+D denominado MapCom (https://mapcom.es/). El primero (CSO2013-47933-C4) reúne, entre 2007 y 2013, a cuatro grupos coordinados y a más de 70 investigadores. El segundo (PGC2018-093358-B-I00) fija sus análisis entre 2014 y 2018 con el concurso de más 20 investigadores de nueve universidades españolas.

Para este artículo hemos filtrado 152 documentos (94 tesis doctorales, 30 artículos publicados en revistas indexadas y 28 comunicaciones a congresos de la Asociación Española de Investigación en Comunicación) cuyo tema principal tiene que ver con la fotografía o el fotoperiodismo. Las búsquedas que se hicieron en las bases de documentos censados y analizados por MapCom, tanto en los títulos como en las palabras clave, fueron "foto", "fotografía" y "fotoperiodismo". Además, se han revisado una a una estas aportaciones para analizar de forma más precisa su objeto de estudio y la metodología aplicada para la investigación.

El análisis bibliométrico implica el inventario y análisis de conjuntos documentales constitutivos de la producción científica a través de estadística y en él se tienen en cuenta una serie de variables que permiten determinar tendencias.

Las principales variables que se analizan en este trabajo tienen que ver con los datos del registro: año de publicación, soporte institucional (universidad, revista), comunidad autónoma y género del autor o autora y los datos del contenido y el abordaje: temáticas, campos, tipos y modalidades de comunicación, técnicas y objetivos.

\section{Resultados}

\subsection{Años de publicación}

Lejos de una primera hipótesis que apuntaba a que el interés investigador de la fotografía y el fotoperiodismo habría ido creciendo con el tiempo, nos encontramos con que la publicación de artículos y tesis doctorales no atiende a una evolución gradual, sino que tiene picos concretos relacionados con proyectos I+D en marcha que incluyen la fotografía como un tema secundario o principal. Así en el año 2013 y 2017, se dieron los dos picos de mayor publicación de artículos, mientras que 2016 fue el año de mayor número de tesis doctorales (una cifra debida a un aumento general del número de tesis doctorales presentadas ante el cambio de plan universitario en España). 


\subsection{Soportes institucionales}

Con respecto a las universidades que respaldan los resultados de la investigación en comunicación de la fotografía tanto a través de tesis doctorales como de artículos de investigación no se observa ningún ranquin significativo, exceptuando que la UCM se coloca a la cabeza en número de tesis doctorales presentadas en estos 12 años (26\% del total). De hecho, se puede observar una participación bastante similar, por ejemplo, en lo que a comunicaciones sobre el tema se refiere, con un cuádruple empate entre la U. Rey Juan Carlos, U. Internacional de la Rioja, U. Jaume I y U. Complutense. Ver Gráfico 1.

\section{Gráfico 1. Comunicaciones sobre fotografía en congresos AE-IC. Universidades primer/a autor/a}

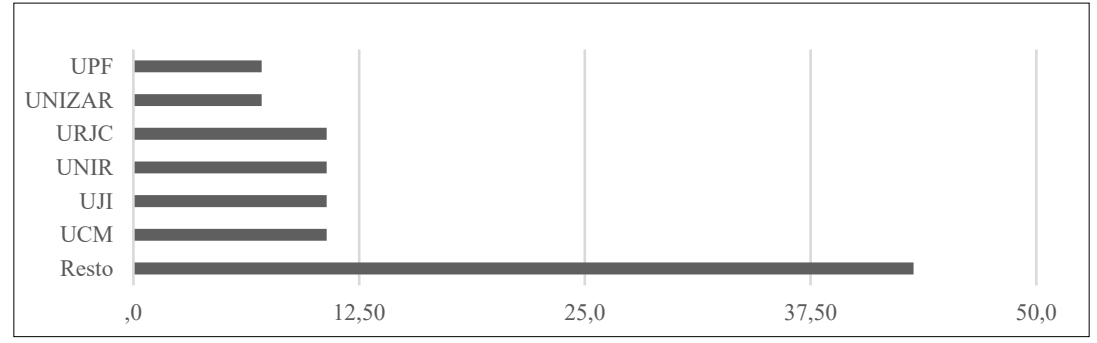

Fuente: Elaboración propia

El soporte revista para los artículos dedicados a la fotografía y relacionados con un proyecto de financiación estatal de I+D tiene un triple empate entre Estudios sobre el Mensaje Periodístico, El Profesional de la Información y Comunicación y Sociedad con 7 artículos cada una $(23,3 \%)$.

\subsection{Perspectiva de género}

La perspectiva de género en la investigación en la comunicación se aplica también a este trabajo de investigación. De ello se extrae que en los inicios de la carrera universitaria, cuando los investigadores juniors presentan sus tesis doctorales poco más de la mitad son doctorandas (52,8\%) mientras que poco menos de la mitad son doctorandos (47,2\%) (Lozano-Ascencio et al., 2020). Sin embargo, cuando se alcanza el grado de doctor se incrementa un declive muy notable en el acceso a cátedra (Caffarel et al., 2018). En lo referente a la investigación en comunicación cuyo objeto de estudio es la fotografía o el fotoperiodismo la diferencia de género es de 6 autoras por 4 autores si tomamos en cuenta los tres documentos analizados (artículos, comunicaciones y tesis doctorales). Ahora bien, existe una paridad exacta en la primera autoría de los artículos a revistas indexadas; en las tesis doctorales 7 de cada 10 son defendidas por mujeres y en las comunicaciones a congresos la primera autoría, en 6 de cada diez casos, la firman los hombres. 


\subsection{Los contenidos de las investigaciones en comunicación sobre la fotografía y el fotoperiodismo}

Tres de cada cuatro documentos analizados tienen una relación directa con el tema central de este artículo (fotografía, periodismo, fotoperiodismo) y el resto se relaciona más con el arte, la publicidad o la propaganda. Algo muy parecido sucede con las tesis doctorales, pues tres de cada cuatro defensas se ciñen al tema principal; el 80\% de los artículos analizados están en la misma línea y dos de cada tres comunicaciones también se centran en la fotografía y el periodismo.

Casi la mitad $(43,4 \%)$ de las temáticas principales de los documentos analizados tienen que ver con las identidades (problemas sociales, emigración, género, conflictos psicológicos); en segundo lugar están las investigaciones que tratan temas relacionados con el oficio y la profesión periodística de la fotografía (empresas, fotógrafos, rutinas profesionales), en última instancia están los temas relacionados con la conceptualización de la fotografía periodística, y la utilización o presencia de las fotografía en internet y redes sociales.

Dentro del Fotoperiodismo, las “identidades" es la principal temática (47 N / 30,9\%), seguida de la "profesión periodística” (36 N / 23,7\%), en tanto que la tercera temática en importancia dentro del Arte, la publicidad y la propaganda también son las "identidades" (19 N / 12,5\%) (Gráfico 2).

Los colectivos más vulnerables de la sociedad -niños, ancianos, mujeres, personas racializadas y el colectivo LGTBIQ+- han sido objeto de interés por parte de las investigaciones centradas en la imagen ofrecida, sobre todo, por los medios de comunicación de masas, y también por las redes sociales.

Las imágenes se relacionan con la construcción de la identidad colectiva y estos trabajos están en consonancia con el auge de los estudios culturales, con especial relevancia a los de perspectiva de género, tanto en el ámbito académico nacional como internacional. A este tipo de investigaciones se asocian también los estudios de descolonización centrados en minorías étnicas o el poder de las culturas predominantes en el mundo.

En las temáticas estudiadas, sorprende que no se hayan trabajado apenas las principales figuras del fotoperiodismo tanto históricas como actuales y que en los trabajos científicos que se dedican a la ética de la profesión periodística sea casi nula la aparición del fotoperiodista, en tanto en cuanto que su desarrollo profesional requiere de unos métodos y códigos específicos; ni la del editor o editora gráfica. 


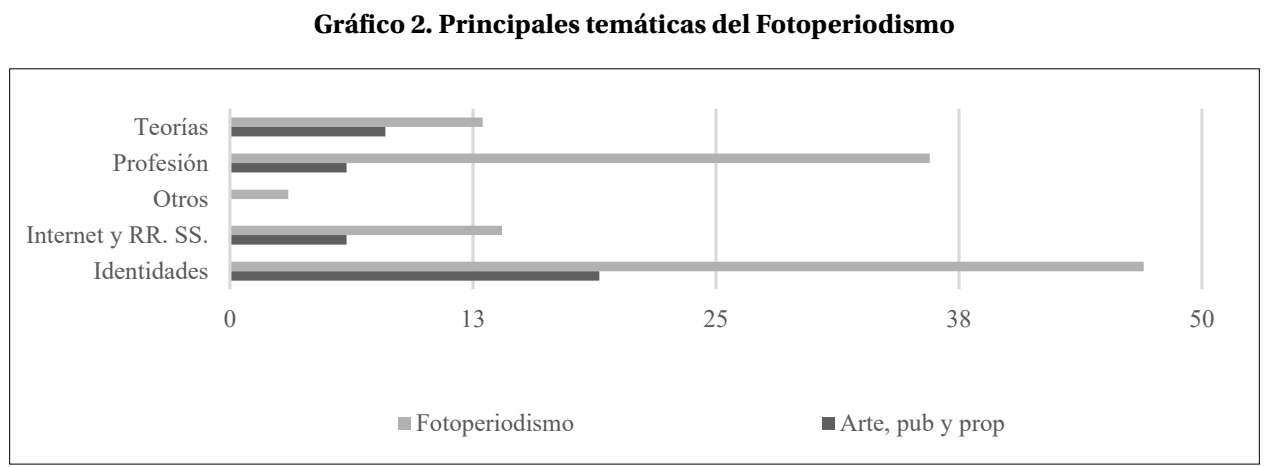

Fuente: Elaboración propia

\subsection{Según el tipo de comunicación}

La mitad de los documentos estudiados (52\%) hacen sus distintos análisis sobre la fotografía tomando en consideración la comunicación a través de los medios de comunicación. Es decir, partiendo de la búsqueda de datos o imágenes en los medios de comunicación. El 48\% restante (73 documentos), colocan sus intereses en la comunicación organizacional, grupal, interpersonal u otros.

Llama la atención que casi la mitad $(51,3 \%)$ de los documentos analizados prefieran localizar las imágenes y documentos de manera impresa, frente al 30,9\% de análisis realizados con imágenes de manera virtual. Gráfico 3.

Gráfico 3. Presencialidad/virtualidad de los Objetos de estudio de la fotografía

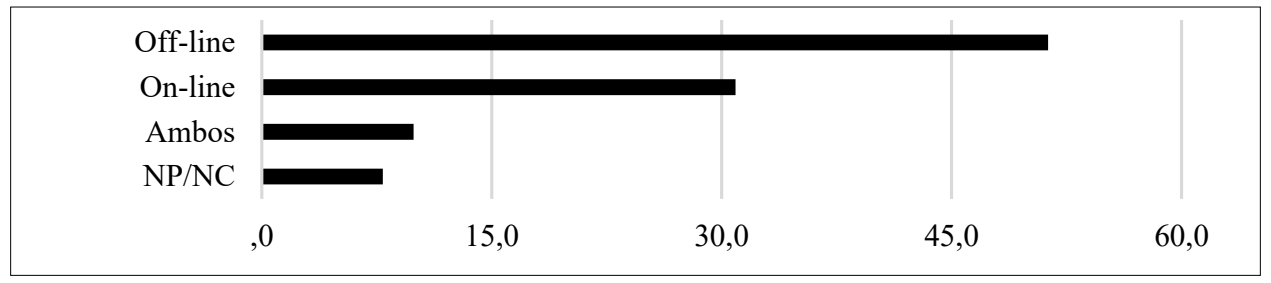

Fuente: Elaboración propia

\subsection{Técnicas de investigación de la fotografía}

La gran mayoría de los documentos analizados $(60,5 \%)$ utiliza técnicas documentales (Análisis de Contenido en revistas, periódicos, en campañas publicitarias y políticas) para abordar sus objetos de estudio y extraer información. El resto de los documentos utilizan técnicas centradas en la observación sistemática o participante en la que se incluyen estudios semióticos $(17,8 \%)$, encuestas $(7,9 \%)$ y entrevistas en profundidad $(7,2 \%)$. 
En el análisis de los datos globales recopilados a través del proyecto MapCom se ha observado que el framing, agenda-setting o encuadre es una de las metodologías en alza en la investigación en comunicación en España. No obstante, el estudio se suele centrar en el análisis de contenido textual, dejando a un lado la interpretación de la imagen que lo acompaña.

Sin embargo, se ha detectado que las técnicas documentales cuantitativas (análisis de contenido), con objetivos descriptivos y centrados en la comunicación mediática, con referencias teóricas del periodismo (framing, agenda-setting o encuadre) no llegan a analizar el material gráfico que suele acompañar a los mensajes. El ámbito científico internacional, sin embargo, se está centrando cada vez más en este aspecto, por lo que suponemos que se trasladará pronto al ámbito nacional un mayor interés científico por este tema.

\subsection{Objetivos de la investigación de la fotografía}

Otro de los puntos a analizar son los objetivos de investigación, que no siempre se expresan directamente en los trabajos de investigación, por lo que se deben interpretar por parte de los analistas implicados en el grupo de investigación. En el Gráfico 4 puede observarse que poco más de la mitad de los documentos $(55,3 \%)$ son descriptivos, es decir, sus procedimientos están orientados a registrar imágenes fotográficas para hacer seguimientos documentales: clasificar el patrimonio artístico, catalogar el papel social de la mujer, medir la información política en campañas electorales, etc. El 27\% de los casos tienen una finalidad explicativa, es decir, exponen causas, efectos, correlaciones y evolución de lo que estudian proponiendo modelos de análisis y simulaciones para el mejor estudio de las imágenes. Por el último el 8,6\% de las investigaciones tienen objetivos de evaluación, es decir, validan, contrastan o ponen a prueba modelos ya existentes para analizar representaciones visuales, construcción de identidades, narraciones documentales o de ficción, etcétera. Llama la atención que muy pocos investigadores (2\%) de las tesis doctorales, artículos indexados y comunicaciones a congresos se hayan planteado la finalidad de intervenir para cambiar conductas o procesos sociales mediante la utilización de dinámicas experimentales aplicadas a individuos aislados o grupos para realizar sus investigaciones. La intervención es una finalidad muy poco frecuente en la mayoría de las investigaciones en comunicación en España.

Gráfico 4. Objetivos de la investigación de la fotografía

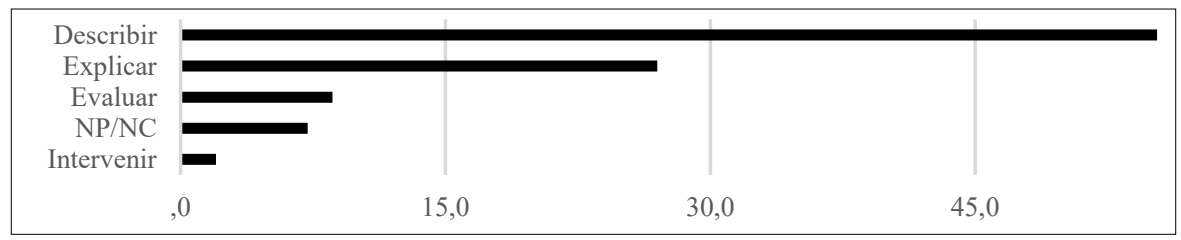

Fuente: Elaboración propia 


\section{Conclusiones}

Las investigaciones de alto rendimiento en España sobre Comunicación entre 2007 y 2018 se ocupan de forma escasa de la fotografía y el fotoperiodismo, pero la mitad de estos documentos muestran líneas de investigación en sintonía con demandas sociales como son los estudios culturales de la identidad.

El estudio de los datos MapCom deja claro que una de las principales debilidades de esta apuesta es la económica y es que, pese al creciente interés social por la fotografía y el fotoperiodismo, éste no se ha traducido aún en un incremento de la producción científica vinculada a proyectos $\mathrm{I}+\mathrm{D}+\mathrm{i}$. Habría que entrar en mayor profundidad sobre las estructuras de solicitantes de este tipo de proyectos y las temáticas elegidas habitualmente para atisbar quizás una de las posibles trabas al desarrollo de la investigación en esta temática no habitual como es el fotoperiodismo y la fotografía y que se asocia a un área de menor prestigio que otras con un minoritario grupo de seguidores y, por lo tanto, un menor impacto. Sin embargo, son tiempos de cambio que reclaman también aires nuevos en la manera de afrontar proyectos I+D+i competitivos y con una clara vocación de transferencia del conocimiento. Desde el momento en el que se impulsen proyectos competitivos cuyo objeto de estudio principal sea la fotografía y el fotoperiodismo, el interés por esta área crecerá y, a la vez, retroalimentará a los estudios de Comunicación y redundará en su prestigio entre la comunidad científica.

Se abre además la puerta de un nuevo profesorado académico vinculado a la cada vez más habitual asignatura de fotoperiodismo en las facultades de comunicación y de ciencias de la información de las universidades españolas que apuesta no solo por un plan docente de corte práctico, sino también teórico, en el que el conocimiento de la historia, así como de las metodologías de análisis de la imagen ocupan un lugar relevante entre las competencias que se prevén conferir al alumnado.

La investigación en fotografía y fotoperiodismo en estos doce años muestra algunos otros signos de debilidad como la falta de una inmersión completa en el estudio de la imagen on-line o de la imagen digital; la incorporación del material gráfico en la aplicación de metodologías como el framing, también conocido como agenda-setting o encuadre; el análisis de los trabajos de fotoperiodistas actuales o históricos; la deontología profesional del fotoperiodista y del editor gráfico y su relación con la era de la posverdad.

Y es en ese mismo punto donde se abre una oportunidad, pues podría ser el momento de innovar metodológicamente dando mayor relevancia a las técnicas centradas en la observación sistemática o participante (en donde se incluyen metodologías como la semiótica y que por el momento solo ocupan el 17,8\% del total de los trabajos estudiados), las encuestas (que por el momento solo representan el 7,9\% de los documentos analizados) y las entrevistas en profundidad (con apenas el 7,2\% del total). Es más, nos sorprende que apenas cuatro trabajos aborden las figuras y los trabajos de fotoperiodistas relevantes a lo largo de la Historia, un vacío que estamos seguros se irá trabajando.

También es relevante destacar que la necesidad hoy en día de trabajar el fact cheking para contrarrestar los efectos de las fake news creadas con imágenes falsas o manipuladas podrá impulsar un modelo de trabajo científico hasta el momento muy poco 
representativo como es el de la intervención para cambiar conductas y procesos sociales. Esta línea de trabajo puede contribuir a la transferencia del conocimiento, pero por ahora apenas representa el $2 \%$ del total de trabajos analizados.

Como hemos ido desgranando, estas debilidades detectadas en nuestra investigación pueden dar a simple vista una imagen de desestructuración de la investigación en fotografía y fotoperiodismo en el ámbito de la comunicación, pero tras un análisis con la puesta en común de diversos factores contextuales, lo que nos muestran son la posible evolución hacia un modelo de investigación transdisciplinar y contemporáneo, en el que la fotografía sea interpretada desde diferentes enfoques analíticos, de manera que no haya una única forma de acercarse a ella, sino que incluso sea un objeto de estudio que requiera de lo interdisciplinar y estar así a la altura de las nuevas formas de pensar y de investigar.

\section{Agradecimientos}

Este artículo ha sido traducido por Brian O’Halloran.

\section{Referencias bibliográficas}

Alcaide, E. (2017). Fotoperiodismo 3.0. Libros.com.

Amores, J. J., Calderón, C. A., \& Stanek, M. (2019). Visual frames of migrants and refugees in the main Western European media. Economics \& Sociology, 12(3), 147-161. https://10.14254/2071-789X.2019/12-3/10

Ardevol-Abreu, A. (2015). Framing o teoría del encuadre en comunicación. Orígenes, desarrollo y panorama actual en España. Revista Latina De Comunicación Social, 70(70), 423-450. https://10.4185/RLCS-2015-1053

Baeza, J. (2000). LEDO, Margarita. Documentalismo fotográfico. Anàlisi: Quaderns De Comunicació i Cultura, , $230-232$.

Baron Pulido, M. (2020). ¿Narrativas para la guerra o para la paz? La fotografía como diacronía periodística. Historia $Y$ Comunicación Social, 25(1), 239-250. https://10.5209/hics.69241

Barthes, R. (2007). La cámara lúcida: nota sobre la fotografía / Roland Barthes (12a ed. ed.). Barcelona etc.: Paidós.

Caffarel, C., Ortega, F., \& Gaitan, J. (2018). La investigación en comunicación en España: Debilidades, amenazas, fortalezas y oportunidades. Comunicar (Huelva, Spain), 26(56), 61-70. https://10.3916/C56-2018-06

Cerdán Martínez, V., \& Padilla Castillo, G. (2019). Historia del “fake” audiovisual: "deepfake” y la mujer en un imaginario falsificado y perverso. Historia Y Comunicación Social, 24(2), 505-520. https://10.5209/hics.66293

Del Prado Flores, R., Moreno Basurto, L. Z., \& Chávez Castañeda, M. (2020). Representaciones fotográficas de la sociedad del cansancio y la pobreza. Revista Latina De Comunicación Social, (75), 291-312. https://10.4185/RLCS-2020-1427

Del Ramo, J. (2016). Análisis de contenido de la representación fotográfica de la crisis de los refugiados sirios y su incidencia en el framing visual. Scire, 22(2), 87-98. 
Fontcuberta, J. (2016). La furia de las imágenes: notas sobre la postfotografía. Galaxia Gutenberg.

García-Ramos, F., \& Jiménez-Gómez, I. (2020). La investigación académica sobre fotografía en España. Un análisis de las tesis doctorales entre 2010 y 2020 . Discursos Fotográficos, 14(43) http://www.uel.br/revistas/uel/index.php/discursosfotograficos/ article/view/42724/pdf

Guallar, J., Codina, L., Freixa, P., \& Pérez-Montoro, M. (2020). Desinformación, bulos, curación y verificación. Revisión de estudios en Iberoamérica 2017-2020. Telos (Maracaibo, Venezuela), 22(3), 595-613. https://10.36390/telos223.09

Leon, Y. C. G., Lara, R. A., \& Rodríguez, L. M. (2018). La construccion fotografica de la realidad internacional en los periodicos nacionales cubanos Granma y Juventud Rebelde/The photographic construction of international reality in the Cuban national newspapers Granma and Juventud Rebelde. Estudios Sobre El Mensaje Periodístico, 24(2), 1251. https://10.5209/ESMP.62213

Lozano-Ascencio, C., Gaitán-Moya, J., Caffarel-Serra, C., \& Piñuel-Raigada, J. (2020). Una década de investigación universitaria sobre Comunicación en España, 2007-2018. El Profesional de la Información, https://10.3145/epi.2020.jul.12

Marzal-Felici, J. (2021). Propuestas para el estudio de las imágenes en la era de la posverdad. Profesional De La Información (EPI), $30(2)$.

Mayoral, J., Parratt, S., \& Morata, M. (2019). Desinformación, manipulación y credibilidad periodísticas: una perspectiva histórica. Historia Y Comunicación Social, 24(2), 395-409. https://10.5209/hics.66267

Sánchez-Vigil, J. M., Salvador-Benítez, A., \& Olivera-Zaldua, M. (2021). Producción científica sobre Fotografía en las revistas de Documentación (2000-2019). Revista Española de Documentación Científica, 44(1), e287. https://10.3989/redc.2021.1.1749

Schwalbe, C. B. (2013). Visually framing the invasion and occupation of Iraq in Time, Newsweek, and US News \& World Report. International Journal of Communication, 7, 24.

Sontag, S. (2007). Sobre la fotografía / Susan Sontag; traducción de Carlos Gardini; revisada por Aurelio Major. Madrid: Alfaguara. stylefix. 